\title{
Characterisation of Malaysian durian (Durio zibethinus Murr) cultivars: relationship of phycochemical and flavour properties with sensory properties
}

\begin{abstract}
The physicochemical ( $\mathrm{pH}$, soluble solids, titratable acidity, sugars and organic acids), flavour and sensory properties of five Malaysian durian cultivars (D2, D24, MDUR78, D101 and Chuk) were studied. There were significant differences $(\mathrm{P}<0.05)$ among the five cultivars in terms of all physicochemical characteristics tested with the exception for D2 and MDUR 78, which had similar physicochemical characteristics. Twenty two esters, 14 sulphur compounds, 7 alcohols, 3 aldehydes and 1 ketone were detected in the durian pulp of the five different cultivars using solid-phase microextraction coupled to gas chromatography-time of flight mass spectrometry. Diethyl disulphide, ethyl-n-propyl disulphide, diethyl trisulphide and ethanethiol were the predominant sulphur-containing compounds in all the cultivars. The major esters present in durian were either ethyl propanoate, ethyl-2-methyl butanoate, or propyl-2-methylbutanoate and their levels varied within cultivars. Principal component analysis applied to the data differentiated all cultivars based on 29 volatile flavour compounds exhibiting significant differences $(\mathrm{P}<0.05)$ between cultivars. Principal components 1 and 2 explained $89 \%$ of the total variance. A strong correlation was observed between sensory properties with flavour compound and physicochemical characteristics of the fruit.
\end{abstract}

Keyword: Durian, Volatile compounds, SPME, GC-TOFMS, Physicochemical, Sensory 